\title{
Effect of adherence to carbohydrate counting on metabolic control in children and adolescents with type 1 diabetes mellitus
}

Sinem Bayram, PhD', Gül Kızıltan, PhD', Onur Akın, $\mathrm{PhD}^{2}$

'Department of Nutrition and Dietetics, Faculty of Health Science, Baskent University, Ankara, Turkey

${ }^{2}$ Department of Nutrition and Dietetics, Gulhane Education and Research Hospital, Faculty of Health Science, Baskent University, Ankara, Turkey
Received: 2 December, 2019

Revised: 18 February, 2020

Accepted: 2 March, 2020

Address for correspondence:

Sinem Bayram, PhD

Department of Nutrition and Dietetics, Faculty of Health Science, Baskent University, Ankara, Turkey

Tel: +90-5335434657

Fax: $+90-3122466666$

E-mail:metins@baskent.edu.tr

https://orcid.org/0000-0003-15697747
Purpose: Carbohydrate counting provides better glycemic control and flexibility than other food planning methods. Consistent adherence to such a complex method is difficult, especially for youth. However, studies that determine adherence to this method and whether it alters metabolic control are limited. The aim of the current study was to determine adherence to this method and investigate its effect on metabolic control, anthropometric measurements, insulin dose, and energy intake.

Methods: In this prospective cross-sectional study, 53 children and adolescents with type 1 diabetes mellitus aged 2 to 18 years and receiving intensive insulin therapy were trained and followed for 6 months. Demographics, anthropometrics, insulin requirements, hemoglobin $\mathrm{A} 1 \mathrm{c}(\mathrm{HbA} 1 \mathrm{c})$, fasting lipids, and food records at baseline and study conclusion were evaluated. At the end of the study patients were divided into adherer and nonadherer groups according to carbohydrate estimate deviations from standardized daily sample menus and calculations for accurate insulin doses. More than 10-g variation in daily consumed carbohydrate amount or failure to decide bolus insulin dose was defined as a nonadherer.

Results: The mean $\mathrm{HbA} 1 \mathrm{c}$, low-density lipoprotein cholesterol, and body mass index standard deviation score changed after the carbohydrate counting training while the mean $\mathrm{HbA} 1 \mathrm{c}$ between groups was significant $(P<0.05)$. Total daily insulin doses increased, and the mean high-density lipoprotein cholesterol levels decreased in both groups. There were significant correlations between $\mathrm{HbA} 1 \mathrm{c}$ and carbohydrate deviation scores as well as $\mathrm{HbA} 1 \mathrm{c}$ and caregiver's education level.

Conclusion: Since adherence to carbohydrate counting may affect metabolic control, health professionals should evaluate and monitor carbohydrate counting skills of caregivers and patients in order to improve efficiency.

Keywords: Type 1 diabetes mellitus, Carbohydrate counting, Adherence, Carbohydrate, Child, Adolescents

\section{Introduction}

Metabolic control is an integral component of diabetes management. ${ }^{1)}$ For the reason that carbohydrates are the principal macronutrient affecting glycemic excursions, intensive insulin therapy and carbohydrate estimation become central to optimizing metabolic control according to the Diabetes Control and Complications Trial. Carbohydrate counting has been reported to provide better glycemic control and flexibility than other meal planning methods in children and adolescents with type 1 diabetes mellitus (T1DM). ${ }^{2)}$ Since the method requires frequent blood glucose monitoring, keeping food records, reading food nutrition labels, and food weighing, adherence associated problems have been reported. ${ }^{3)}$ 
Children and adolescents have different age-specific hemoglobin Alc (HbAlc) goals than adults who require special standards of care. Many children and adolescents have elevated postprandial glucose levels and $\mathrm{HbAlc}$ values that exceed age-specific goals. ${ }^{3)}$ Common barriers to children and adolescent adherence are peer influences, depression, disordered eating, insufficient continuous glucose monitoring, and so on. Moreover, such cognitive and emotional factors affect metabolic control and quality of the diet. ${ }^{4)}$ Previous studies indicate low adherence to dietary guidelines in type 1 diabetic children and adolescents. ${ }^{5-8)}$ To prevent obesity, dyslipidemia, hypertension and the microvascular complications associated with hyperglycemia, dietary adherence and quality should be improved. Thus, individual counseling, regular training, and more intensive education are needed for both patients and their families. $^{3,9)}$

Some patients think they are applying "carbohydrate counting" however they estimate preprandial insulin doses with approximate estimation rather than calculating carbohydrate content of the meal. Thus, they can over- or underestimate a meal's carbohydrate content. ${ }^{10)}$ Accurate estimation of carbohydrates to be consumed in a meal is critical to achieving target glycemic control. Although no standardized approach to assess adherence to carbohydrate counting is available, carbohydrate estimation and quantity of carbohydrate consumed in relation to insulin dose can be examined. ${ }^{5,6)}$ It is well known that carbohydrates are primary postprandial glucose modifying macronutrients. ${ }^{9)}$ Smart et al. ${ }^{11)}$ have proven that $20 \mathrm{~g}$ of ingested glucose causes significant postprandial hyperglycaemia when insulin is not given. Based on this result 20 -g variation in actual carbohydrate content can critically affect postprandial glycaemia in children using intensive insulin therapy. Thus, researchers stated that carbohydrate estimations should be within $10 \mathrm{~g}$ of the actual meal carbohydrate for optimal postprandial glucose.

Few data exist on adherence to carbohydrate counting in youth with T1DM, yet it is a recommended part of diabetes management. ${ }^{12,13)}$ Bolus insulin adjustments for the meal are associated with optimal glycemic control independent of which method is used when assessing carbohydrate amount. However, the impact of carbohydrate counting on serum lipid parameters and BMI $z$-scores are not yet clear. Consequently, the aim of this study was to assess the effect of adherence to carbohydrate counting on glycemic control, serum lipid levels, anthropometric measurements, total daily insulin dose, and energy intake among children and adolescents with T1DM.

\section{Materials and methods}

\section{Subjects}

After exclusion of patients with another systemic disease except T1DM and those that were using medications that interfere with diabetes management, 53 children and adolescents followed by the Gulhane Education and Research Hospital Pediatric Endocrinology Department and ages 2 to 18 years, receiving intensive insulin therapy, and using carbohydrate counting for meal planning for at least 6 months were enrolled in the study. For analyses, 5 patients who did not attend follow-up visits or had missing records were excluded, thus 48 patients' results were evaluated in the final analysis.

\section{Study plan}

Sociodemographic information, nutritional habits, and disease related data were collected via a survey. The survey was completed at the time of the routine clinic visit, and diet records were completed in a week after the visit.

Problems associated with the carbohydrate counting method were determined by the dietitian through preliminary interviews and education repeated for confused patients. All interviews (hospital visits and phone calls) were conducted by the same researcher.

\section{Dietary intake and nutrient analysis}

Dietary habits were examined using an open ended, dietitianadministered dietary history in a section of the survey. Families were instructed to keep detailed 24-hour dietary recall since it has been reported to capture dietary intake with less bias than food-frequency questionnaires. Recalls were performed on 3 consecutive days in a week, including 2 weekdays and 1 weekend. A photographic atlas was used to record the type and portion size of the food/fluid/meal. Dietary records were checked by the dietitian and asked to be repeated if needed.

The energy and nutrient intakes of patients were analyzed by the dietitian using the Nutrition Database for Turkey Ebispro for Windows (BEBIS), Germany, version 7.0 $0^{14)}$ and compared with the Recommended Dietary Allowances (RDA). ${ }^{15)}$ BEBIS is a software program that is designed to calculate the nutritional values of Turkish foods and commercial foods.

\section{Anthropometrical measurements}

Height was measured to the nearest $0.1 \mathrm{~cm}$, and weight to nearest $0.1 \mathrm{~kg}$ in light clothing and without shoes. All anthropometrical measurements were performed by the same trained staff. Body mass index (BMI) and BMI standard deviations (SDs) according to age and sex were calculated and evaluated with World Health Organization child growth references and standards. According to these, -1 to +1 SD is accepted as normal weight, $<-1$ SD as underweight, +1 to +2 as overweight and $\geq+2$ SD as obese. ${ }^{16)}$

\section{Carbohydrate counting training}

Individual training was conducted for study participants. On the first day, general information such as healthy meal planning, 
the effect of nutrients (carbohydrate, protein, fat) on glycemia, the amount of carbohydrate in foods, foods containing $15 \mathrm{~g}$ of carbohydrate defined as a carbohydrate change, and portion sizes were taught by solving sample menus. For this training, a 4-hour interview was conducted with each patient. At the end of this phase, patients were asked to record their fasting and postprandial blood glucose measurements, insulin doses, nutrient consumption, and carbohydrate content of their meals for the next day. On the second and third days these records were checked, and subjects such as meal planning and frequency; interaction between consumed foods, physical activity, and insulin doses; and correct label reading according to the type of insulin used by the patient were discussed. Target blood glucose levels were determined followed by discussions about how to achieve them.

It was thought that advanced carbohydrate counting training could be initiated in patients whose blood glucose control was achieved and whose basal insulin dose was adjusted correctly. After endocrinologists reported that participating patients met this condition, the patients were recalled a week later with their 5 -day food records, at least 4 fasting and postprandial blood glucose measurement records, and the form on which they recorded insulin doses. Meal-specific carbohydrate/insulin ratios were calculated according to the amount of carbohydrates consumed in patients' meals and changes in blood glucose levels. The patients were taught how to use the insulin sensitivity factor (IDF). Although the duration of the training varied according to the patients' level of understanding, a 4-hour interview was conducted and this stage of the training was repeated in those who could not answer the questions correctly. After the 3 stages of carbohydrate counting were taught, the training was completed by dissolving samples with groups of 3 to 4 patients and their parents so that patients could share their knowledge and experiences. Participation in group trainings varied depending on the patient's/family's desire and level of understanding.

\section{Adherence evaluation}

All 3-day food records and standardized daily sample menus were evaluated to determine their accuracy in carbohydrate estimation. The mean difference between patients' and dietitians' carbohydrate estimation was computed. To set adherence classification, $\geq 10$-g variation from actual carbohydrate content (dietitian assessment) of meals was used. The carbohydrate content of snacks and its accuracy was not included for the adherence evaluation because patients did not cover snacks with bolus insulin.

All patients and parents were asked to calculate the carbohydrate content of standardized daily sample menus and their own food records to define how they would intervene for low, normal, and high blood glucose levels in the carbohydrate count adherence form in order to determine the compliance and skills of carbohydrate counts at the end of the sixth month. Questions asked on the carbohydrate count adherence form included, "Did the patient administer the correct insulin dose according to the menu consumed," "Did the patient calculate the carbohydrate content appropriately," and "Did the patient use the IDF correctly?"

\section{Biochemical parameters}

$\mathrm{HbAlc}$ and lipid parameters were used to evaluate metabolic control. $\mathrm{HbAlc}$ values were evaluated according to goals of The Society of Turkish Endocrinology and Metabolism of Turkey $(<7.5 \%-8.5 \%$ for ages $<6$ years old, $<8.0 \%$ for ages 6 to 13 years old, and $6.5 \%-7.0 \%$ for ages $13-19$ years old) ${ }^{17)}$ Reference values of the American Academy of Pediatrics Clinical Report on Lipid Screening in Children were used for lipid parameter classifications. ${ }^{18)}$ According to this report, levels of low-density lipoprotein (LDL)-cholesterol $<110 \mathrm{mg} / \mathrm{dL}$, high-density lipoprotein (HDL)-cholesterol $\geq 35 \mathrm{mg} / \mathrm{dL}$, total cholesterol $<170$ $\mathrm{mg} / \mathrm{dL}$, and triglycerides $<160 \mathrm{mg} / \mathrm{dL}$ were accepted as reference values.

\section{Statistical analysis}

Data analysis was conducted using the statistical software package IBM SPSS Statistics software ver. 22.0 (IBM, Armonk, New York, USA). Continuous variables are presented as the mean and SD, and categorical variables as the frequency and percentage. Fisher exact test was used for independent group comparisons in categorical variables. Variables were examined for normality distribution by the Shapiro-Wilk test. If the distribution was normal, comparisons between baseline and final values were performed with the paired sample t-test; otherwise, the Wilcoxon signed-rank test was used. The independent samples $t$-test or Mann-Whitney test was used to compare the mean difference between groups. Pearson correlation coefficients were utilized to determine correlation values between variables. Significant values of $P<0.05$ were considered to be statistically significant.

\section{Results}

The mean age of study group participants $(n=53)$ was $10.8 \pm 4.0$ years and the interquartile range of diabetes duration was $0.79-4.62$ years. Demographic, clinical, laboratory, and general dietary characteristics of the adherer and nonadherer patients at baseline are given in Table 1 . There was no significant difference between the 2 groups at the beginning of the study $(P>0.05)$.

No changes in hypoglycemic episodes were observed. At the beginning of the study the mean hypoglycemia prevalence in a month was $6.91 \pm 7.43$ times, at the end of the study it was $6.75 \pm 6.31(P>0.05)$. Although the mean values of $\mathrm{HbAlc}$ did not differ during the study period for both adherer and nonadherer groups, it differed between adherer and nonadherer 
groups within the study time $(P<0.05)$. Daily total insulin doses increased and total energy consumption decreased after 6 months in both groups $(P<0.05)$. Metabolic and anthropometric changes in the adherer and nonadherer groups during the study are given in Table 2.

Correlations between carbohydrate deviation score and some parameters are given in Table 3. According to Table 3, there was a positive correlation between $\mathrm{HbAlc}$ and insulin doses $(P<0.05)$. On the other hand, BMI-standard deviation score positively correlated with insulin doses and LDL. Caregiver's eduction level (CEL) correlated negatively with $\mathrm{HbAlc}$ and insulin doses $(P<0.05)$. There was a significant positive correlation between carbohydrate deviation score (CDS) and HbAlc.

Carbohydrate counting affects significant change in insulin dose and energy regardless of adherence. Adherence affects the

Table 1. Demographic, clinical and laboratory characteristics of adherer and nonadherer groups at baseline

\begin{tabular}{lccc}
\hline Variable & Adherer & Nonadherer & P-value \\
\hline $\begin{array}{l}\text { No. of patients } \\
\text { Sex }\end{array}$ & $27(50.9)$ & $26(49.1)$ & \\
$\quad \begin{array}{l}\text { Female } \\
\text { Male }\end{array}$ & $14(51.9)$ & $16(61.5)$ & 0.353 \\
Age (yr) & $13(48.1)$ & $10(38.5)$ & \\
$\begin{array}{l}\text { Diabetes duration } \\
\text { (mo) }\end{array}$ & $10.64 \pm 4.40$ & $10.53 \pm 3.71$ & 0.927 \\
BMI SDS & $24.59 \pm 5.71$ & $28.81 \pm 3.52$ & 0.102 \\
HbA1c (\%) & $0.49 \pm 0.93$ & $0.52 \pm 0.72$ & 0.879 \\
Total insulin dose & $7.85 \pm 1.53$ & $8.23 \pm 1.53$ & 0.404 \\
(U/kg/day) & $0.75 \pm 0.25$ & $0.83 \pm 0.25$ & 0.352 \\
TG (mg/dL) & $85.88 \pm 37.83$ & $104.17 \pm 73.75$ & 0.280 \\
TC (mg/dL) & $175.48 \pm 31.99$ & $168.69 \pm 27.75$ & 0.438 \\
HDL-C (mg/dL) & $55.72 \pm 13.74$ & $56.17 \pm 6.31$ & 0.885 \\
LDL-C (mg/dL) & $102.68 \pm 25.33$ & $93.47 \pm 23.02$ & 0.196 \\
\hline Vlues are presented as number & & \\
\hline
\end{tabular}

Values are presented as number (\%) or mean \pm standard deviation. BMI, body mass index; SDS, standard deviation (z) score; LDL-C, low-density lipoprotein cholesterol; HDL-C, high-density lipoprotein cholesterol; TG, triglycerides; TC, total cholesterol. significant decrease in $\mathrm{HbAlc}$. HbAlc was positively correlated with CDS and negatively correlated with CEL.

\section{Discussion}

T1DM is a disease with high morbidity and mortality due to acute and chronic complications that cause growth retardation in children because of varying benefits from nutrients. $\beta$ cell damage is very rapid in T1DM, especially at young ages. Carbohydrate counting is a meal planning method that allows better glycemic control due to the advantages of enabling individual freedom and continuation of social life. ${ }^{12)}$ It is very difficult to plan and adapt nutritional therapy in children and adolescents with special needs. In this study, the researchers aimed to increase adherence by increasing the frequency and awareness of nutritional education.

Little is known about the ability of children to count carbohydrates and whether a particular method for assessing carbohydrate quantity is better than others. This study investigated how accurately children and their caregivers estimated the carbohydrate content of a sample menu, and based on results, researchers divided the children into adherer or nonadherer groups. Demographic and metabolic profiles of adherer and nonadherer children were similar at the beginning of the study. Adherence to carbohydrate counting in children with T1DM revealed that approximately $51 \%$ adhered to this method. In a similar study conducted in Brazil, adherence to the prescribed diet was reported as $54.2 \% .{ }^{19)}$ In a study that reviewed current findings on dietary adherence in youth with T1DM, rates of adherence to eating behaviors ranged from $21 \%-95 \%$.

The metabolic control parameter (HbA1c) of nonadherer children increased by the end of the study when compared to baseline $(P<0.05)$, however, no such difference was determined in other selected metabolic control parameters. The HbAlc levels were not significantly different after the intervention, although the adherer group had lower HbAlc levels within the

Table 2. Metabolic and anthropometric changes in the adherer and nonadherer groups during the study

\begin{tabular}{|c|c|c|c|c|c|c|c|c|c|}
\hline \multirow{3}{*}{ Variable } & \multicolumn{9}{|c|}{ Carbohydrate counters $(n=53)$} \\
\hline & \multicolumn{3}{|c|}{ Adherer $(n=27)$} & \multicolumn{3}{|c|}{ Nonadherer $(n=26)$} & \multicolumn{3}{|c|}{ Change from baseline } \\
\hline & Baseline & 6th month & $P$-value & Baseline & 6th month & $P$-value ${ }^{\ddagger}$ & Adherer & Nonadherer & $P$-value \\
\hline $\mathrm{HbA1c}(\%)$ & $7.85 \pm 1.53$ & $7.63 \pm 0.95$ & 0.364 & $8.23 \pm 1.22$ & $8.73 \pm 1.47$ & 0.060 & $-0.22 \pm 1.05$ & $0.50 \pm 1.36$ & $0.043^{*}$ \\
\hline LDL-C (mg/dL) & $102.68 \pm 25.33$ & $99.00 \pm 22.33$ & 0.469 & $93.47 \pm 23.02$ & $91.34 \pm 20.02$ & 0.628 & $-3.00 \pm 24.50$ & $-2.13 \pm 21.04$ & 0.816 \\
\hline $\mathrm{HDL}-\mathrm{C}(\mathrm{mg} / \mathrm{dL})$ & $55.72 \pm 13.74$ & $53.64 \pm 11.25$ & 0.340 & $56.17 \pm 6.31$ & $54.78 \pm 11.17$ & 0.487 & $-2.08 \pm 12.05$ & $-1.39 \pm 8.72$ & 0.319 \\
\hline Insulin dose $(\mathrm{U} / \mathrm{kg} / \mathrm{d})$ & $0.81 \pm 0.53$ & $0.87 \pm 0.61$ & $0.009^{*}$ & $0.95 \pm 0.52$ & $1.15 \pm 0.67$ & $0.041^{*}$ & $0.06 \pm 0.58$ & $0.20 \pm 062$ & 0.285 \\
\hline BMI-SDS & $0.49 \pm 0.93$ & $0.40 \pm 1.01$ & 0.210 & $0.52 \pm 0.72$ & $0.51 \pm 0.71$ & 0.827 & $-0.49 \pm 0.95$ & $-0.01 \pm 0.71$ & 0.476 \\
\hline Height-SDS & $0.31 \pm 0.89$ & $0.32 \pm 0.61$ & 0.845 & $0.38 \pm 0.67$ & $0.39 \pm 0.51$ & 0.735 & $0.01 \pm 0.78$ & $0.01 \pm 0.63$ & 0.375 \\
\hline Weight-SDS & $0.21 \pm 0.90$ & $0.18 \pm 0.75$ & 0.310 & $0.43 \pm 0.87$ & $0.42 \pm 0.85$ & 0.875 & $-0.03 \pm 0.83$ & $-0.01 \pm 0.87$ & 0.957 \\
\hline Energy (kcal/kg) & $54.63 \pm 26.38$ & $45.33 \pm 21.02$ & $0.004^{*}$ & $52.80 \pm 19.38$ & $45.62 \pm 14.55$ & $0.001^{*}$ & $-9.30 \pm 25.05$ & $-7.18 \pm 16.09$ & 0.555 \\
\hline
\end{tabular}

Values are mean \pm SD.

HbA1C, Hemoglobin A1C; LDL-C, low-density lipoprotein cholesterol; HDL-C, high-density lipoprotein cholesterol; BMI, body mass index; SDS, standard deviation (z) score.

${ }^{*} P<0.05$. ${ }^{\dagger}$ Comparison of mean differences between adherer and nonadherer group within study time. ${ }^{\ddagger}$ Comparison of baseline and 6 th month values within in each group. 
Table 3. Correlations between carbohydrate deviation score and education level of caregiver, energy intake, BMI-SDS, some metabolic control parameters

\begin{tabular}{|c|c|c|c|c|c|c|c|}
\hline Variable & LDL & $\mathrm{HDL}$ & Insulin doses & BMI-SDS & Energy & CEL & CDS \\
\hline $\mathrm{HbA1c}$ & $-0.118(0.416)$ & $0.094(0.517)$ & $0.084(0.045)^{*}$ & $-0.007(0.962)$ & $0.087(0.557)$ & $-0.370(0.010)^{*}$ & $0.427(0.002)$ \\
\hline LDL & & $0.295(0.134)$ & $0.027(0.706)$ & $0.294(0.041)^{*}$ & $0.064(0.667)$ & $0.061(0.693)$ & $-0.145(0.327$ \\
\hline $\mathrm{HDL}$ & & & $0.059(0.602)$ & $0.045(0.757)$ & $-0.122(0.410)$ & $0.097(0.525)$ & $0.092(0.534)$ \\
\hline Insulin doses & & & & $0.560(0.004)^{*}$ & $0.240(0.480)$ & $-0.450(0.023)^{*}$ & $0.068(0.340)$ \\
\hline BMI-SDS & & & & & $0.037(0.803)$ & $0.001(0.997)$ & $0.020(0.895)$ \\
\hline Energy & & & & & & $0.041(0.790)$ & $0.086(0.562)$ \\
\hline CEL & & & & & & & $0.267(0.080)$ \\
\hline
\end{tabular}

Values are Pearson correlation coefficient (P-value).

BMI, body mass index; SDS, standard deviation (z) score; LDL, low-density lipoprotein; HDL, high-density lipoprotein; HbA1c, hemoglobin A1c; CEL, caregiver's education level; CDS, carbohydrate deviation score.

Difference between patient's and dietitian's carbohydrate estimation.

${ }^{*} P<0.05$.

study time. It is well known that even a modest reduction in HbAlc level decreases the risk of microvascular complications. ${ }^{2)}$ One study reported that although metabolic control ( $\mathrm{HbAlc})$ was good in patients with T1DM, only one-third of patients were able to maintain postprandial normoglycemia and $40 \%$ experienced frequent hyperglycemia. This drawback leads researchers to search for different methods. Conventional carbohydrate counting (focusing on carbohydrate content) is the most often used insulin carbohydrate matching method because of the proven effectiveness and safety of carbohydrate counting, and therefore there is limited literature on other methods (protein-fat counting, etc.). ${ }^{20)}$

In the present study, the highest $\mathrm{HbAlc}$ levels were found in children whose caregiver was less educated and who failed to estimate the carbohydrate content of the sample menus. The caregiver's level of education is important since the caregiver carries out the calculations of the carbohydrate counting, especially in young children. Consistent with these results, the International Society for Pediatric and Adolescent Diabetes Clinical Practice Guidelines stated that family, especially the primary caregiver's education level, has an significant role in accomplishing carbohydrate counting. ${ }^{21)}$

A careful counting of carbohydrates will lead to the correct calculation of the required insulin dose, which in turn will lead to normalizing postprandial glycemia. This should only be achieved by accurate carbohydrate counting techniques. The results of studies conducted with adolescents are controversial in that carbohydrate counting affects the total daily insulin dose. $^{22-25)}$ In this study insulin doses increased after training in both groups. One of the reasons for this increment could be the rapid growth of children and adolescents during the study time. Another reason may be incorrect calculation of the required insulin doses. Most adolescents consume more saturated fat and animal protein and less fiber regardless of T1DM. It is well known that it is carbohydrates that primarily affect glycemia. However, it should be remembered that high consumption of protein and fat is critical for deciding the correct insulin dose.

In both groups LDL and HDL cholesterol levels were among the normal values recommended by the American Academy of Pediatrics clinical report on lipid screening in children. ${ }^{18)}$ This may be because both groups received carbohydrate count training and therefore increased healthy nutrition mindfulness. Studies determining macronutrient intakes revealed higher than recommended intake of fat and saturated fat and lower intake of fruits, vegetables, and whole grains in youth with T1DM. ${ }^{5)}$ As dieticians increase their knowledge and skills about carbohydrate counting, the situation can be reversed.

The results of studies conducted with children and adolescents applying carbohydrate counting regarding changes in body weight are contradictory; while some studies have reported an increase, ${ }^{26-28)}$ others have reported a decrease ${ }^{10,29)}$ and yet others have reported no change. ${ }^{1,24)}$ In the current study, similar BMI $z$-scores in adherer and nonadherer groups were found both before and after training.

Young children with T1DM may be at increased risk for dietary adherence due to aspects of food preferences, food refusal, emotional lability, and behavioral resistance. ${ }^{30}$ Adolescence is also marked by feelings of ambivalence, impulsiveness, and mood swings; the struggle to separate from parents; and the need to be accepted by peers; therefore, dietary adherence is reduced in this period.9)

The dietician's responsibilities in T1DM education should be to explain the importance of a nutrition plan, the types of carbohydrates and their effects on glycemia, why refined carbohydrates should be avoided, the role of proteins and fats in glycemia, and the importance of fiber consumption for patients and their parents. Patients should understand the relationship between insulin, nutrition, and exercise after carbohydrate counting training. In addition, the dietician should emphasize what should be considered in meal planning, out-of-home nutrition, shopping, and food preparation and cooking, and provide information about sweeteners and dietary products. Nutritional education and lifestyle changes should be individualized in a patient-centered manner. Maintaining healthy eating behaviors, providing optimum glycemic control, reducing cardiovascular risk factors, preserving psychosocial health, and maintaining family dynamics should be the basic strategies of nutrition therapy. ${ }^{9,31)}$ Most importantly, these 
trainings should be repeated periodically, and quizzes should be administered to patients about their carbohydrate counting skills.

The follow-up duration for the current study was 6 months, which can be considered as a limitation of the study. The study found that adherence does not currently affect metabolic parameters outside of $\mathrm{HbAlc}$, but is considered to be effective over a long period of time. The effect of dietary adherence on blood lipid parameters can be determined if longer follow-up is planned. Another limitation of the study was the number of patients. The researchers conducted a single-center study to standardize the biochemical parameters and recruited all study patients with T1DM who agreed to participate. An experimental study can be conducted in which all meal consumption and insulin usage of patients are observed because in this study food records were taken based on patients' statements.

In conclusion, nutritional therapy for diabetes is complicated, and numerous studies have shown problems with dietary adherence in patients with T1DM. ${ }^{5,6)}$ However, no study has specifically examined the dietary adherence of young children and adolescents with T1DM and attempted to relate this prospectively to children's metabolic control (glycemic control as well as serum lipid profiles and anthropometrics). Additional studies have determined the effects of diet adherence or carbohydrate counting on metabolic control, however, this study has important and valuable clinical significance for determining the effect of adherence to carbohydrate counting on metabolic control. Adherence training should be regularly provided by a diabetes dietitian in order to achieve good metabolic control in this young group.

\section{Ethical statement}

This study was approved by the Baskent University Institutional Review Board and Ethics Committee (Project number: KA13/218). Informed consent was obtained from the caregivers of all patients as well as themselves if the age $(\geq 7)$ was appropriate.

\section{Conflict of interest}

No potential conflict of interest relevant to this article was reported.

\section{Acknowledgments}

This study was supported by the Baskent University Research Fund.

\section{References}

1. Bouillon R, Carmeliet G. Vitamin D insufficiency: 1. Gökșen D, Atik Altınok Y, Ozen S, Demir G, Darcan S.
Effects of carbohydrate counting method on metabolic control in children with type 1 diabetes mellitus. J Clin Res Pediatr Endocrinol 2014;6:74-8.

2. Anderson EJ, Richardson M, Castle G, Cercone S, Delahanty L, Lyon R, et al. Nutrition interventions for intensive therapy in the Diabetes Control and Complications Trial. The DCCT Research Group. J Am Diet Assoc 1993;93:76872.

3. Spiegel G, Bortsov A, Bishop FK, Owen D, Klingensmith GJ, Mayer-Davis EJ, et al. Randomized nutrition education intervention to improve carbohydrate counting in adolescents with type 1 diabetes study: is more intensive education needed? J Acad Nutr Diet 2012;112:1736-46.

4. Borus JS, Laffel L. Adherence challenges in the management of type 1 diabetes in adolescents: prevention and intervention. Curr Opin Pediatr 2010;22:405-11.

5. Patton SR. Adherence to diet in youth with type 1 diabetes. J Am Diet Assoc 2011;111:550-5.

6. Nansel TR, Haynie DL, Lipsky LM, Laffel LM, Mehta SN. Multiple indicators of poor diet quality in children and adolescents with type 1 diabetes are associated with higher body mass index percentile but not glycemic control. J Acad Nutr Diet 2012;112:1728-35.

7. Patton SR, Dolan LM, Powers SW. Dietary adherence and associated glycemic control in families of young children with type 1 diabetes. J Am Diet Assoc 2007;107:46-52.

8. Rovner AJ, Nansel TR. Are children with type 1 diabetes consuming a healthful diet?: a review of the current evidence and strategies for dietary change. Diabetes Educ 2009;35:97-107.

9. Silverstein J, Klingensmith G, Copeland K, Plotnick L, Kaufman F, Laffel L, et al. Care of children and adolescents with type 1 diabetes: a statement of the American Diabetes Association. Diabetes Care 2005;28:186-212.

10. Laurenzi A, Bolla AM, Panigoni G, Doria V, Uccellatore A, Peretti E, et al. Effects of carbohydrate counting on glucose control and quality of life over 24 weeks in adult patients with type 1 diabetes on continuous subcutaneous insulin infusion: a randomized, prospective clinical trial (GIOCAR). Diabetes Care 201 1;34:823-7.

11. Smart CE, King BR, McElduff P, Collins CE. In children using intensive insulin therapy, a 20 -g variation in carbohydrate amount significantly impacts on postprandial glycaemia. Diabet Med 2012;29:e21-4.

12. Bishop FK, Maahs DM, Spiegel G, Owen D, Klingensmith GJ, Bortsov A, et al. The carbohydrate counting in adolescents with type 1 diabetes (CCAT) study. Diabetes Spectr 2010;22:56-62.

13. Smart CE, Ross K, Edge JA, King BR, McElduff P, Collins CE. Can children with Type 1 diabetes and their caregivers estimate the carbohydrate content of meals and snacks? Diabet Med 2010;27:348-53.

14. Ebispro for Windows, Turkish version Bebis [CDROM]. Version 7.2. Stuttgart: Germany; 2011. Data bases: Bundeslebenmittelschlüssel, II.3 and other sources. 
15. Institute of Medicine. Dietary reference intakes for energy, carbohydrate, fiber, fat, fatty acids, cholesterol, protein, and amino acids. Washington, DC: The National Academies Press, 2005.

16. de Onis M, Onyango AW, Borghi E, Siyam A, Nishida C, Siekmann J. Development of a WHO growth reference for school-aged children and adolescents. Bull World Health Organ 2007;85:660-7.

17. TEMD Diabetes Mellitus Study Group. Diagnosis, treatment and monitoring guideline of diabetes mellitus and its complications [Internet]. Türkiye Endokrinoloji ve Metabolizma Derneği; 2014 [cited 2019 Sep 11]. Available from: http://temd.org.tr/admin/uploads/tbl_ kilavuz/20180814161019-2018tbl_kilavuz6c373c6010.pdf.

18. Horsley L. Practice guidelines American Academy of Pediatrics clinical report on lipid screening in children. Am Fam Physician 2009;79:703-5.

19. Davison KA, Negrato CA, Cobas R, Matheus A, Tannus L, Palma CS, et al. Relationship between adherence to diet, glycemic control and cardiovascular risk factors in patients with type 1 diabetes: a nationwide survey in Brazil. Nutr J 2014;13:19.

20. Bell KJ, Barclay AW, Petocz P, Colagiuri S, Brand-Miller JC. Efficacy of carbohydrate counting in type 1 diabetes: a systematic review and meta-analysis. Lancet Diabetes Endocrinol 2014;2:133-40.

21. Smart CE, Annan F, Bruno LP, Higgins LA, Acerini CL; International Society for Pediatric and Adolescent Diabetes. ISPAD Clinical Practice Consensus Guidelines 2014. Nutritional management in children and adolescents with diabetes. Pediatr Diabetes 2014;15 Suppl 20:135-53.

22. Mehta SN, Volkening LK, Quinn N, Laffel LM. Intensively managed young children with type 1 diabetes consume high-fat, low-fiber diets similar to age-matched controls. Nutr Res 2014;34:428-35.
23. Overby NC, Flaaten V, Veierød MB, Bergstad I, Margeirsdottir HD, Dahl-Jørgensen K, et al. Children and adolescents with type 1 diabetes eat a more atherosclerosisprone diet than healthy control subjects. Diabetologia 2007;50:307-16.

24. Dias VM, Pandini JA, Nunes RR, Sperandei SL, Portella ES, Cobas RA, et al. Effect of the carbohydrate counting method on glycemic control in patients with type 1 diabetes. Diabetol Metab Syndr 2010;2:54.

25. Anderson BJ, Vangsness L, Connell A, Butler D, GoebelFabbri A, Laffel LM. Family conflict, adherence, and glycaemic control in youth with short duration Type 1 diabetes. Diabet Med 2002;19:635-42.

26. Alemzadeh R, Palma-Sisto P, Parton E, Totka J, Kirby M. Beneficial effects of flexible insulin therapy in children and adolescents with type 1 diabetes mellitus. Acta Diabetol 2003;40:137-42.

27. Benhamou PY, Garnier C, Debaty I, Rueff A, Gilbert C, Ressel M, et al. Basal insulin dose in 40 type 1 diabetic patients remains stable 1 year after educational training in flexible insulin therapy. Diabetes Metab 2010;36:369-74.

28. Gunn D, Mansell P. Glycaemic control and weight 7 years after Dose Adjustment For Normal Eating (DAFNE) structured education in type 1 diabetes. Diabet Med 2012;29:807-12.

29. Hayes RL, Garnett SP, Clarke SL, Harkin NM, Chan AK, Ambler GR. A flexible diet using an insulin to carbohydrate ratio for adolescents with type 1 diabetes - a pilot study. Clin Nutr 2012;31:705-9.

30. Wysocki T, Huxtable K, Linscheid TR, Wayne W. Adjustment to diabetes mellitus in preschoolers and their mothers. Diabetes Care 1989;12:524-9.

31. Tascini G, Berioli MG, Cerquiglini L, Santi E, Mancini $\mathrm{G}$, Rogari F, et al. Carbohydrate counting in children and adolescents with type 1 diabetes. Nutrients 2018;10:109. 